\title{
Advancing the mission of cardiovascular molecular imaging
}

\author{
Sharmila Dorbala, MD, MPH, , ${ }^{\mathrm{a}, \mathrm{e}}$ Wunan Zhou, $M D,{ }^{\mathrm{b}, \mathrm{c}}$ and Mehran Sadeghi, MD, \\ $\mathrm{PhD}^{\mathrm{d}}$ \\ a Cardiovascular Division, Department of Medicine, Heart \& Vascular Centre, Brigham and \\ Women's Hospital, Harvard Medical School, Boston, MA \\ b National Heart, Lung and Blood Institute (NHLBI), National Institutes of Health, Bethesda, MD \\ c Cardiovascular Branch, NHLBI, Bethesda, MD \\ d Yale School of Medicine, New Haven, CT \\ e Division of Nuclear Medicine and Molecular Imaging, Department of Radiology, Brigham and \\ Women's Hospital and Harvard Medical School, Boston, MA
}

Received Dec 9, 2020; accepted Dec 9, 2020

doi: 10.1007/s12350-021-02534-9

Molecular imaging is critical to advance the future of nuclear cardiology and personalized medicine. The ability to assess physiology of in vivo systems makes molecular imaging techniques uniquely positioned to improve disease diagnosis, prognostication and treatment. Despite these advantages and the exciting recent advances in molecular imaging, knowledge of cardiovascular (CV) molecular imaging is not widely disseminated. Meaningful academic interactions between molecular cardiologists and cardiovascular molecular imagers are needed to catalyze innovation. The lack of forums for global scale interactions between these experts, may be one important but addressable barrier. Thus, taking advantage of the interlude in laboratory work worldwide during the peak of the COVID19 pandemic, ASNC has launched a new course-ASNC Virtual CV Molecular Imaging Seminars.

The goals of these seminars were to (1) promote the value of $\mathrm{CV}$ molecular imaging in research and patient care (2) provide a unique platform for research collaborations in CV molecular imaging and (3) engage trainees in incorporating molecular imaging and nuclear cardiology in their early career development. These Virtual CV Molecular Imaging Seminars attracted CV imaging trainees and faculty from 27 countries around

Reprint requests: Sharmila Dorbala, MD, MPH, Cardiovascular Division, Department of Medicine, Heart \& Vascular Centre, Brigham and Women's Hospital, Harvard Medical School, Boston, MA ; sdorbala@bwh.harvard.edu

J Nucl Cardiol

$1071-3581 / \$ 34.00$

Copyright (C) 2021 American Society of Nuclear Cardiology. the globe to interact with internationally acclaimed investigators and their laboratory researchers.

Each week, the seminars started with a didactic session on biology and disease pathophysiology, followed by research presentations by trainees from various molecular imaging laboratories. The format was highly interactive with an expert discussant promoting discussion through real- time attendee questions. Rising stars in molecular imaging research were invited to serve as session moderators. Through these sessions, molecular imaging researchers and trainees interacted with each other virtually and learned from each other. The forum was conducive to virtual networking with global leaders in molecular cardiology and CV molecular imaging. The broad topics discussed in these seminars included Atherosclerosis, New Technologies in Nuclear Cardiology, Vascular Calcification, Transthyretin Cardiac Amyloidosis, Cardiovascular Inflammation, Coronary Microvascular dysfunction. The spring seminars concluded at the end of June. The next series of seminars were reinitiated in the Fall starting with an NIH Seminar on September 23rd, 2020. If your laboratory is interested in participating in these seminars, please reach out to the course organizers listed below.

To continue the momentum set forth by the Series and engage the broader nuclear cardiology community, Dr. Ami Iskandrian, Editor, Journal of Nuclear Cardiology (JNC), has started a new series: Molecular Imaging Corner in JNC. The faculty/trainees who presented their research were invited to submit a brief review summarizing their molecular imaging research. We are excited that the first of those is being published in this issue of JNC and look forward to future reviews: 
Course Organizers:

Sharmila Dorbala, sdorbala@bwh.harvard.edu

Mehran Sadeghi, mehran.sadeghi@yale.edu

Wunan Zhou, wunan.zhou@nih.gov

Table. Topics covered in the virtual molecular imaging seminars.

\section{Topic}

1 Atherosclerosis- Part 1

2 Atherosclerosis- Part 2

3 New Technologies in Nuclear Cardiology: Part 1

4 New Technologies in Nuclear Cardiology: Part 2

5 Vascular Calcification

6 Transthyretin Amyloidosis

7 Cardiovascular inflammation

8 Coronary microvascular dysfunction 1

9 Coronary microvascular dysfunction 2
Publisher's Note Springer Nature remains neutral with regard to jurisdictional claims in published maps and institutional affiliations. 